\title{
Identidades desterritorializadas. El sentimiento de pertenencia nacional entre los adolescentes de familias inmigradas
}

\author{
Eduardo Terrén $(\dagger)$ \\ Universidad de Salamanca. Departamento de Sociología y Comunicación
}

Recibido: 30-03-2008

Aceptado: 01-07-2008

\section{Resumen}

La integración identitaria es una dimensión ya reconocida en los tratamientos tanto académicos como institucionales de la integración de la población inmigrada. El caso de las comúnmente denominadas «segundas generaciones» constituye un terreno de investigación específico en el que analizar los procesos culturales implicados en esta dimensión identificacional de la integración. El trabajo aquí presentado se enmarca en un proyecto de investigación más amplio que estudia precisamente los procesos de adaptación cultural experimentados por los adolescentes procedentes de familias inmigradas. Una de las dimensiones abordadas en este estudio ha sido el sentimiento de pertenencia nacional que se observa en esta población. La metodología utilizada se basa en el análisis transversal de las más de noventa entrevistas recogidas por el proyecto. El caso más saliente lo constituyen los pertenecientes a la generación 1,5 . Lo que el análisis de sus relatos biográficos revela es que su identificación nacional con el país de acogida es más bien débil y ambivalente respecto a la identificación con el origen, y que, en todo caso, es generalmente más latente que manifiesta. El análisis conduce a la discusión de dos ideas generales: por un lado, que los procesos de hibridación esperados a partir de un marco teórico transnacional o postcolonial parecen menos manifiestos de lo que cabía esperar y, por otro, que las resistencias observadas a la identificación nacional con el destino pueden estar muy relacionadas con el rechazo al proyecto migratorio de la familia.

Palabras clave: integración cultural; juventud inmigrante; identidad nacional.

Abstract. Deterritorialized Identities. The sense of national belonging among teenagers proceeding from immigrated families

The identificational integration is a dimension already recognized in the both academic and institutional treatments of the integration of the immigrated population. The «second generation» within immigrant families constitutes a specific area of research in which to analyze the cultural processes involved in this dimension of integration. This paper arises from a wider project of research on the cultural adjustment experienced by teenagers proceeding from immigrated families. One of the aspects approached in this study has been the feeling of national belonging. The article is based on the transversal analysis of more than ninety interviews gathered by the project. The analysis of these biographical statements shows that their national identification with the country of reception is rather weak and ambivalent with regard to the identification with the origin. This leads to the discussion of two general 
ideas: on the one hand, that the processes of hybridization expected from a postcolonial or transnational theoretical frame seem to be less manifest than what was expected; on the other hand, that the observed resistances to the national identification with Spain might be closely related to the rejection of the migratory project of the family.

Key words: cultural integration; immigrant youth; national identity.

\section{Sumario}

1. Marco y objeto de la investigación

2. Un repaso al estado de la cuestión

3. El bricolaje de la pertenencia: camino seguido y discusión de resultados
4. Conclusiones provisionales

Referencias bibliográficas

\section{Marco y objeto de la investigación}

Los movimientos migratorios están modificando como nunca la estructura demográfica de España, pero también su estructura cultural. El impacto de estos flujos y de los stocks de población que generan se suman al de las fuerzas de la globalización, que, a través de los nuevos derroteros de la movilidad de personas e informaciones, están alterando radicalmente el sentido de la diversidad cultural (Terrén, 2007) y de las formas de ciudadanía. Uno de los principales efectos de este impacto es la posible transformación del mapa de sentimientos cívicos y, en especial, entre todos ellos, de los que tienen que ver con la pertenencia y la vinculación a un territorio determinado. Si es cierto que la capacidad de integración de una sociedad es directamente proporcional a la diversidad que es capaz de albergar (Terrén, 2003: 263), el modo como se desarrollen estos sentimientos de pertenencia en la identidad política y social de los hijos de familias inmigradas es parte esencial del futuro de nuestra cultura política como sociedad de acogida. Sin embargo, lo cierto es que, hoy por hoy, nuestro conocimiento de cómo se produce este proceso de incorporación cívica en el caso de los hijos de los inmigrantes es todavía muy limitado. El trabajo que aquí se presenta pretende contribuir a expandir dicho conocimiento.

La pertenencia nacional ha venido siendo el sentimiento cívico por excelencia; el soporte tradicional de la vinculación comunitaria en las sociedades modernas (Rex, 1996). No obstante, su naturaleza es todo menos clara, pues su significado es difícilmente separable de las creencias de quienes lo albergan (Miller, 1997). Por eso, inicialmente, es necesaria una breve discusión preliminar del concepto que, a pesar de mostrar su dificultad inherente, nos permita hacernos con una definición manejable del concepto y mostrar la pertinencia de su estudio científico en relación con el fenómeno migratorio.

Desde la perspectiva de quienes entienden la identidad nacional en términos de pertenencia cultural (Miller, 1997 y 2000; Poole, 1999), partimos de la base de que el sentimiento de pertenencia nacional proporciona $-\mathrm{O}$, al menos, 
ha proporcionado hasta ahora ${ }^{1}$ - un cierto cemento cohesivo que favorece la integración social de sociedades constituidas por poblaciones relativamente estables sobre un mismo territorio. Más durkheimianamente, podríamos decir que es un alimentador de conciencia colectiva porque es fuente de identificaciones, compromisos y lealtades que constituyen la clave del ejercicio de la ciudadanía, más allá de su estatus legal o jurídico. En suma, el sentimiento de pertenencia nacional ha sido proveedor durante varios siglos de modernidad de esa mínima vinculación comunitaria de la que precisa toda sociedad. Ha sido, así, una de esas «ideas principales» en torno a las que los ciudadanos «deben reunir su juicio», según decía ya Tocqueville (1980) en su estudio de la entonces incipiente república norteamericana.

Tradicionalmente, la pertenencia nacional se ha entendido como una noción sólida y robusta, y asociada tanto a la homogeneidad cultural como a una análoga concepción compacta de la ciudadanía. Ambas se han edificado sobre un mismo paquete de componentes jurídicos, culturales y territoriales férreamente vinculados. De esta vinculación compacta podría decirse lo mismo que desde el ya clásico trabajo de Benedict Anderson (1983) se viene diciendo de la nación en sí misma, esto es: que se trata de una identidad inventada; una construcción histórica y no una esencia inmutable; sólo una y una muy peculiar (aunque extendida y arraigada) forma de revestir fronteras; de asociar el territorio, los privilegios de quienes están arraigados en él y la cultura normalmente vinculada a una lengua.

La conciencia nacional es un fenómeno relevante para el estudio de la integración cultural, pues ésta se construye en gran parte a partir del ejercicio de derechos ligados a los sentimientos de pertenencia a una comunidad política y cultural (es decir, dotada de instituciones y simbólicamente constituida) que se define como nacional. Sin embargo, la creciente movilidad de las personas cuestiona las bases tradicionales sobre las que se ha entendido la pertenencia nacional. La profusión de identidades múltiples, a la vez que de nuevas y porosas formas de adscripción, ha llevado a pensar que quizá la fuente básica de la pertenencia ya no pueda encontrarse en la comunidad nacional y a sentir la necesidad de buscar fórmulas de pertenencia más flexibles (Castles y Davidson, 2000: VIII $)^{2}$. Ésta es la apuesta que resulta de la proliferación de casos como el que ilustra un joven trabajador entrevistado para esta investigación:

1. Digo que es o ha venido siendo porque, frente a las propuestas que hablan efectivamente de una nueva "ciudadanía postnacional» (véanse las referencias de la nota 2), deben tenerse en cuenta opiniones como las de Lucas (2001: 105), para quien, a la hora de construir un vínculo político, hemos enterrado con excesiva precipitación a la pertenencia nacional cuando no contamos todavía con un sustituto eficaz que la reemplace como comunidad de pertenencia.

2. La reflexión sobre las consecuencias de las nuevas formas de inclusión (y exclusión) de no nacionales en la conceptualización de la ciudadanía se plasma en diferentes categorizaciones, como la «ciudadanía transnacional» (Bauböck, 1994), la "ciudadanía multicultural» (Kymlicka, 1995), la "ciudadanía diferenciada» (Young, 1990), la "ciudadanía neorrepublicana» (Van Gusteren, 1994), la «ciudadanía cultural» (Turner, 1993) o los «miembros postnacionales» (Soysal, 1994). 
Pienso quedarme a vivir aquí en España, pero yo soy cubano. Eso lo tengo muy claro. [¿Español?] ¡Para nada! Por el carnet de identidad y porque vivo aquí. Pero no me siento español para nada, o un hijo de inmigrantes rusos aún en secundaria y desde hace tres años en España. No seré un español nunca. Puedo tener el carnet y ser español para comprar un coche, una casa, para casarme..., pero yo seré siempre ruso.

Afirma Bauböck $(2004,2006)$ que el movimiento de individuos cruzando fronteras lleva consigo un desvanecimiento de las mismas (crossing/blurring), pero esta idea apunta sobre todo a las fronteras físicas, y es demasiado simple para mostrar la interrelación que existe entre las migraciones y las fronteras simbólicas, aquellas que nutren $-\mathrm{y}$, al mismo tiempo, se nutren- de sentimientos cívicos como el de la pertenencia nacional. Una relación más compleja entre crossing y blurring puede parecer paradójica, pero es, sin duda, empíricamente más plausible. Por curioso que pueda parecer, el hecho es que — siguiendo lo señalado por Saassen respecto a la economía y la política-, mientras que la globalización económica y cultural desnacionalizan las economías nacionales y las fuentes de información, las migraciones —elemento sustancial de esa globalización - parecen contribuir en sus efectos a la renacionalización de muchos de los sentimientos cívicos que integran la cultura política de las sociedades de acogida. De hecho, difícilmente puede negarse que, aunque, como ya hemos señalado, el incremento de los flujos migratorios de las últimas décadas ha llevado a un cuestionamiento de la noción tradicional de la ciudadanía basada en la nacionalidad, también ha coincidido igualmente con un reforzamiento de los nacionalismos y las identidades locales.

Lo cierto es que la construcción y la reconstrucción de las identidades es uno de los fenómenos que más se ve afectado por los procesos migratorios (Segura, 2006; Fukuyama, 2007). No en vano, hoy día constituye ya una dimensión básica que complementa la integración económica, social y cultural con los inmigrados. La forma en que los individuos se piensan a sí mismos se ve sometida a tensiones específicas cuando ese pensamiento se pone en marcha en contextos en los que los grupos, los valores o las costumbres divergen de los que constituyen las referencias habituales (cercanas o íntimas). El conflicto de adaptación cultural que se vive entonces se conoce como aculturación, y una de las formas de estudiarlo es a través de la producción o transformación de las identidades colectivas de los individuos implicados en el movimiento migratorio y, muy concretamente, a través de su sentimiento de pertenencia nacional, que constituye una de las fuentes de esa identidad colectiva (Schwartz, Montgomery y Briones, 2006). El interés de fondo de este estudio radica, pues, en saber en qué medida las expresiones empíricamente constatables de esta pertenencia contribuyen a la «deconstrucción» o «desagregación» (Benhabib, 2005) de esa forma tradicional de entender la ciudadanía nacional que anteriormente hemos comentado.

Las cuestiones que se debaten en el espacio de esta "deconstrucción" pueden articularse en torno a dos ejes de reflexión y análisis. Uno, de carácter más 
bien normativo, se centra en la pertinencia de la condición de nacional de un estado como requisito para el disfrute de los derechos asociados al estatus de ciudadanía ${ }^{3}$. Otro, de corte más empírico, se centra en el análisis de cómo se transforman, en este mundo globalizado, las identidades políticas y culturales locales (si, por ejemplo, tienden a difuminarse y a fundirse, o si tienden a afianzarse y radicalizarse o si, más bien, tienden a transformarse y a redefinirse en un nivel más abstracto).

Es en este segundo eje en el que se encuadra la investigación que aquí se presenta y las reflexiones que origina. Su objetivo general es contribuir al entendimiento de cómo se relacionan los descendientes de inmigrantes con la narrativa de la identidad nacional. La pregunta general a la que pretende contribuir es: ¿cómo puede incorporarse la diversidad cultural y el complejo proceso que se describe en la aculturación de los hijos de los inmigrantes a la reconstrucción de la identidad nacional en un mundo globalizado?

\section{Un repaso al estado de la cuestión}

El estudio de la construcción de la identidad en los adolescentes adquirió madurez antes en la psicología social que en la sociología propiamente dicha, sobre todo desde que Erik H. Erikson (1992) vinculó inseparablemente ambas cuestiones, al considerar la consecución de una autoidentidad saludable como el objetivo central de esta etapa biográfica. Para Erikson, la identidad que se obtiene a partir de las respuestas producidas a la pregunta por excelencia ("¿Quién soy yo?») está indisolublemente unida a las preferencias y a los compromisos que se establecen con entidades colectivas (Goodenow y Espin, 1993). Pero la investigación más específica sobre el desarrollo del sentimiento de pertenencia nacional fue abierta en los años cincuenta por Piaget y Weil (1951), en una línea muy centrada en la evolución del desarrollo cognitivo y en las categorías de conocimiento necesarias para emitir juicios sobre la nacionalidad o los extranjeros. Hoy día parece haberse impuesto un renovado interés por estas cuestiones (Barrett, Riazanova y Volovikova, 2001; Bennett, Lyons, Sani y Barrett, 1998; en España: Torres, 1994; Vila et al., 1998; Molero, 1999), pero más sobre la estela de la psicología social desarrollada en los años sesenta y setenta (Jahoda, 1964; Middleton et al., 1970).

No se encuentra en esta literatura un interés específico por el caso de los adolescentes procedentes de familias inmigrantes, pero ello no quita para que puedan extraerse de ella tres enseñanzas relevantes para nuestro objeto. La primera es que, como pone de manifiesto la investigación comparativa más espe-

3. Estas discusiones están normalmente asociadas a propuestas teóricas como la de la «estratificación cívica» de Morris (2002), la de la conocida jerarquía de Martiniello (1994): full citizens, denizens y margizens, o la de la "desnacionalización de la ciudadanía" propuesta por Velasco (2006). Pieza estelar de estos debates es el concepto de «identidad postnacional» de Habermas - un tipo de identidad política no basado en una tradición cultural nacional, sino referido al ejercicio de los derechos políticos y al «patriotismo constitucional». 
cífica sobre el desarrollo de las identificaciones nacionales en los adolescentes, no parece del todo plausible hoy el tipo de universalismo que, desde Piaget, había dominado el estudio del desarrollo mental sobre las categorías sociales en niños y adolescentes. Efectivamente, la investigación transcultural tiende a mostrar que apenas existen procesos universales (excepción hecha del favoritismo hacia el in-group nacional desde los seis años). Barret (2001), por ejemplo, ha mostrado que las actitudes de los adolescentes hacia la nacionalidad dependen enormemente del entorno cultural en que crecen y no pueden explicarse exclusivamente a partir de un desarrollo cognitivo básico y universal, como ocurre en buena parte de la investigación sobre el desarrollo de las bases cognitivas del prejuicio y las percepciones sociales. La segunda enseñanza es que la identificación nacional está generalmente ligada, además, a ciertos tipos de motivación, es decir, tiende a afirmarse más o a dirigirse hacia aquellos objetos con cuya vinculación se obtiene una mayor autoestima y un mejor autoconcepto (Lyons, 1996; Spinner-Halev y Theiss-Morse, 2003). La tercera, íntimamente relacionada con lo anterior, es que, en cualquier caso, y al igual que ocurre en general con las categorías morales y las representaciones sociales, el estudio de las identificaciones nacionales sugiere que, junto al plano de lo cognitivo, existe también un plano afectivo (de forma que son los sentimientos de pertenencia, los compromisos emocionales y las valoraciones diferenciales y preferencias respecto de lo propio y lo extraño lo que condiciona el sentido de las categorías cognitivas con las que se piensan) (Barrett, 2001).

Desde el punto de vista de la sociología de las migraciones, el interés por la identidad de los hijos de los inmigrantes está ligado al curso de su integración en las sociedades de acogida ${ }^{4}$. No obstante, la cuestión de la pertenencia nacional no ha sido suficientemente destacada y ha tendido a ser subsumida en la investigación sobre la identidad étnica y los procesos de aculturación. Y es que, efectivamente, uno de los objetos de investigación más salientes en el estudio sociológico de las denominadas "segundas generaciones» ha sido el de su adaptación cultural, a caballo entre el mundo que pervive en la familia y el que se percibe fuera de ella. Desde esta perspectiva, el estudio de las actitudes de pertenencia ha estado normalmente vinculado al del desarrollo de la identidad étnica 5 .

Sobre este aspecto, existe un amplio consenso en que, dentro del complejo mapa de dificultades que constituye la adolescencia, el caso de los adolescentes descendientes de inmigrantes tiene dimensiones específicas, pues estos adolescentes se enfrentan a complejas cuestiones de adaptación a la hora de lidiar con las identidades étnicas o raciales de sus progenitores y con las percepcio-

4. Suele hablarse, en este sentido, del campo de las «segundas generaciones», pero no son infrecuentes las críticas a una categoría - muchas veces estigmatizante- que proyecta sobre los descendientes lo que, en realidad, es condición de los prognitores (García Borrego, 2003, 2004; Massot, 2003).

5. Como apunta Miller (1997), existe una clara intersección entre la identidad étnica y la nacional, pero conviene distinguirlas analíticamente. 
nes sociales que la sociedad tiene de los mismos, lo que a menudo les lleva a aprender a desenvolverse en ambientes muy diferenciados con habilidades diversas (Waters, 1996, Rumbaut, 1994; Phinney, 1990). Muy frecuentemente, estos adolescentes viven entre dos mundos poco coincidentes, lo que les lleva en ocasiones a experimentar un peculiar conflicto intergeneracional y un fuerte estrés de aculturación (Kibria, 2002; Rumbaut, 1994; Zhou, 2001), pero también a construir las autoidentificaciones étnicas de forma muy maleable y a vivir con gran fluidez las fronteras entre grupos (Portes y MacLeod, 1996).

La hipótesis intuitivamente más esperable y dominante todavía en la etnosociología de la migración es la que se corresponde con lo que en la literatura sobre las llamadas «segundas generaciones» se conoce como el «modelo lineal» de la asimilación cultural, una perspectiva forjada en buena medida sobre la experiencia de los primeros inmigrantes de origen europeo a los EE. UU. a comienzos del siglo XX. La idea era (es) que cuanto más tiempo lleve el inmigrante en la sociedad de acogida, más aumenta su exposición y la de sus descendientes a la cultura dominante y, en consecuencia, más probable es que los adolescentes procedentes de estas familias rompan los vínculos y las identificaciones con los padres y adopten un sentimiento de pertenencia nacional que les identifique con la sociedad de acogida. A largo plazo, la idea canónica del straight-line model subyacente a esta teoría clásica de la asimilación era que, en definitiva, cuanto más tiempo pasa, la progresiva aculturación se traduce en una mayor asimilación cultural y una consiguiente disolución, pérdida de vitalidad o mera reducción a vestigio simbólico de la identidad y distintividad de la primera generación. Por lo que a la identidad respecta, esta teoría clásica sobre la asimilación de los inmigrantes en las sociedades de acogida preveía unos procesos sucesivos de aculturación en la lengua y las normas de la mayoría que terminarían, al cabo de varias generaciones, por producir como subproceso fundamental de la asimilación una identificational assimilation (Gordon, 1964) que afectaría igualmente a los sentimientos cívicos. De esta manera, si se acepta que el sentimiento de pertenencia nacional forma parte de la identidad étnica y cultural de un individuo, lo esperable a partir de esta tesis clásica es que los individuos descendientes de inmigrantes mostraran una progresiva identificación con la pertenencia al país de acogida.

Sin embargo, investigaciones recientes realizadas desde la perspectiva del capital social y el transnacionalismo (Levitt y Glick Schiller, 2004; Glick Schiller y Fouron, 2001; Vertovec, 2003; Portes, Guarnizo y Landolt, 1999) han cuestionado los supuestos de la teoría asimilacionista de la aculturación lineal. Por lo que respecta a la adaptación cultural de los descendientes de inmigrantes, esta línea de investigación ha mostrado repetidamente que los modelos lineales no se cumplen necesariamente y que, en realidad, muchas familias inmigrantes - y, sobre todo sus hijos- pueden llegar a desarrollar trayectorias positivas de incorporación manteniendo, y no necesariamente renunciando a, sus identidades de origen (aunque tampoco, en realidad, éstas siguen siendo exactamente las de origen). Para algunos de estos estudios, conservar una determinada identidad con arreglo a una comunidad étnica o inmigrante puede 
facilitar enormemente en algunos casos la incorporación a la sociedad de destino (Portes y MacLeod, 1996; Waters, 1999; Zhou y Bankston, 1998).

En esta línea de investigación se ha mostrado repetidamente, durante la última década, que la conservación de sentimientos de identidad intensamente relacionados con los países de origen o con las comunidades étnicas recreadas en destino no sólo se mantiene mientras dura el envío de remesas o mientras dura la inseguridad de la llegada y se precisan con urgencia redes de apoyo. Lo que ocurre, contra lo sostenido por la tesis clásica, es que, con el tiempo, dichos sentimientos y actividades no se van diluyendo necesariamente en proporción directa a la integración de los inmigrantes en la sociedad de acogida. Ocurre más bien que esos vínculos no se debilitan sin más, ni se pierden entre los que llevan más tiempo o entre sus descendientes; más bien se mantienen, sólo que con intensidades y componentes muy variables. Así es que el panorama de la continuidad cultural y afectiva con las identidades de origen es mucho más compleja de lo que parecía: no necesariamente viven más transnacionalmente quienes viven más aferrados a comunidades étnicas en destino, ni viven más así sólo quienes terminan de llegar, ni los patrones que se observan en algunos grupos en unos lugares se reproducen sin más en otros (Portes, 2003; Morawska, 2003, 2004; Faist, 2004; Levitt, 2003). No sólo porque se ha revelado enorme la variabilidad de formas adaptativas que exhiben las identidades puestas en juego por los descendientes de los inmigrantes, sino también porque lo que ha tomado cuerpo con ellos es un proceso de elaboración de la pertenencia que es complejo en sí mismo y que no puede reducirse fácilmente a cierres categoriales definitivos. Una mediadora de origen marroquí nos lo contó así:

Nunca me han considerado de allí, nunca he sido, a los ojos de los demás y de la familia, nunca he sido marroquí, siempre he sido «la de fuera». Aquí, no de niña, pero ya de mayor, siempre se me ha considerado extranjera. Así que, realmente, no tengo una identidad, no sé de dónde soy. Mucha gente no entiende que pueda ser de los dos sitios. Me piden que me defina, tú eres de Marruecos o tú eres de España. Bueno, yo considero que soy de los dos sitios, pero ningún sitio considera que soy de ahí, entonces..., lo dejo así.

\section{El bricolaje de la pertenencia: camino seguido y discusión de resultados}

Como contribución al conocimiento empírico de la adaptación cultural de los adolescentes de familias inmigradas, este trabajo quiere profundizar en la construcción de su sentimiento de pertenencia nacional explorando cuál es su relación con algunas variables que se han tenido por fundamentales en la literatura revisada y en las preguntas de investigación tenidas en mente. Por ejemplo: ¿se expresa como una mezcla de identificaciones étnicas o mundos culturales diversos? ¿Se produce de diferente forma en los chicos que en las chicas? ¿Muestra diferencias según la zona de procedencia de la familia? ¿Tiene que ver con la experiencia de traslado y llegada o el hecho de haber nacido en España? ¿Se 
produce de forma diferente según se haya experimentado o no discriminación racial o en función del origen?

Las respuestas encontradas a estas preguntas se basan en el análisis de 93 entrevistas seleccionadas de entre las realizadas en el marco de un proyecto de investigación más amplio y construidas sobre el modelo del método biográfico (Riemann, 2003; Chamberlayne et al., 2000) ${ }^{6}$.

Lo primero que se destaca en el análisis de las narrativas recogidas es el lugar y la forma de presentación del tópico que nos interesa. Claramente, se aprecia que la identificación nacional no es una preocupación central ni ocupa un lugar preferente entre las cuitas de los adolescentes entrevistados. Tratándose de un modelo de entrevista muy sensible a la iniciativa discursiva del entrevistado, es descable que en ningún caso la cuestión surgió de forma espontánea. Cuando el relato lo trató como resultado de una inflexión requerida, las primeras presentaciones tendieron a ser bastante ambivalentes en su mayoría y sólo a resultas de un requerimiento ulterior adoptaron una posición más definida.

Los elementos afectivos que rodean esta identificación la hacen muy compleja en su formulación, porque normalmente se vincula a sentimientos como la vergüenza, el resentimiento o, simplemente, la culpa. Por eso no es infrecuente que, en los circunloquios discursivos que rodean a la declaración, aparezcan matizaciones que a veces se convierten en contradicciones y reconocimientos, tanto de lo que uno cree que es aunque no lo sienta, como de lo que uno siente aunque no crea que lo es. Un hijo de ecuatorianos con diecisiete años y cuatro de residencia en España contaba, por ejemplo, tras haberse referido a los latinos como «los míos»:

Yo tengo nacionalidad española, ya..., ya, sí, ya español. Pero yo todavía me siento muy..., no sé..., sí y por más que me avergüence y por más que diga yo...: «Ah, tronco que vergüenza me da», tengo que reconocer que tengo sangre, o sea..., que yo... soy uno de ellos. Aunque quiera decir que no, soy uno de ellos, aunque yo sea distinto a ellos, aunque me comporte yo de otra manera que ellos... Pero vamos, que soy como ellos, soy uno de ellos, soy de allí...

6. El proyecto de referencia es Identidades en construcción: La adaptación cultural de los adolescentes procedentes de familias inmigradas, MEC, Plan I+D, SEJ 2004-2007. Los sujetos entrevistados tenían entre quince y diecinueve años y eran todos hijos de, al menos, un progenitor inmigrante. Para este trabajo, se han seleccionado 94 entrevistas de la base disponible (en el proyecto, se entrevistó también a adolescentes que habían emigrado solos, MENAS o mayores de edad sin padres inmigrantes, pero no los consideramos en este análisis), distinguiendo entre ellos, por un lado, a los que habían nacido en España o habían sido traídos antes de los diez años (la segunda generación propiamente dicha) (27) y, por otro lado, a los que fueron traídos a España después de esta edad, es decir, en su preadolescencia (los conocidos como generación 1,5 o generación intermedia), que fueron los más representados (67). Esta sobrerrepresentación puede explicarse por la dificultad de encontrar todavía individuos de segunda generación comprendidos en el tramo de edad considerado. 
Podría interpretarse si acaso esta aparente irrelevancia de la identificación nacional, e incluso su presentación a veces confusa, puede asociarse con una inmadurez patológica o algún tipo de déficit. En este sentido, parece importante no entender de forma peyorativa esta indefinición como propia de una identidad insana. En otro lugar (Terrén, 2007), advertimos ya contra la tendencia a juzgar la cuestión desde el paternalismo, el adultocratismo o la patologización de la psicología inmigrante. Empuja a ello la habitual asociación de lo confuso o indeterminado con lo insano, algo que ya se apunta en la propia conceptualización de Erikson (1992) y sus seguidores cuando hablan de fases difusas o de moratoria de la identidad, y que es frecuente encontrar en trabajos sobre adolescentes procedentes de la inmigración cuando asocian la identidad «sana» con la "conseguida» o "cerrada». Como ya se dijo anteriormente, hay estudiosos que ya han advertido sobre la necesidad de actualizar la visión continuista y lineal eriksoniana y adaptarla al curso de identidades más fragmentadas y flexibles (Suárez-Orozco y Suárez Orozco, 2003). El hecho de que, al menos en nuestra muestra, no existan diferencias significativas en la acometida discursiva de la cuestión en función de la edad, no avala tampoco el que esa ambivalencia o indefinición inicial pudiera explicarse por una cierta inmadurez asociada a la temprana edad.

Parece, más bien, que se trata de un resorte identitario latente que se activa cuando se aborda en la narración un episodio que asocia lo familiar con el origen o cuando se produce o se percibe una situación de conflicto, bien en origen, bien en destino.

Lo primero es manifiesto cuando se constata en el hecho de que el hogar familiar pesa muy frecuentemente como «recordatorio identitario», lo que muchas veces se resume en expresiones del tipo "cuando llego a casa». A un joven colombiano, por ejemplo, su casa le hacía

[...] sentirme más colombiano aquí [en España] que allí [...], pues mi mama tiene así para colgar las llaves que pone Colombia, hay fotos de nosotros con la bandera de Colombia así o cositas así o, por ejemplo, entras a la cocina y encuentras un Vilo, que es como Cola-Cao, o encuentras una botella de gaseosa de Costobon.

Y, análogamente, una joven cubana de generación 1,5 con diecisiete años y cinco de residencia en España, decía:

Me doy cuenta de que existe esa diferencia porque voy por la calle y me siento una española más; pero cuando llego a casa y oigo a mi madre hablar, y veo lo que..., las cosas que hacemos en casa todos los días, pues..., ¿a quién quieres engañar?

La asociación de estas reflexiones con la imagen del «llegar a casa» subraya, en cualquier caso, el fuerte componente afectivo que encierra siempre este tipo de identificación. 
Sobre lo segundo, conviene recordar que existen ya otros estudios que han mostrado que, aunque la identificación nacional no fuera relevante inicialmente, lo ha terminado siendo una vez producido un contacto significativo con la población mayoritaria o con otros emigrantes en destino. Por ejemplo, Kibria (2002) muestra cómo inmigrantes que, a su llegada a EE. UU., sólo se percibían a sí mismos en términos locales, comenzaron a describirse en términos nacionales (como «chinos» o «italianos») cuando se vieron percibidos como tales. Lo mismo han mostrado Portes y MacLeod (1996) respecto al uso de categorías panétnicas o supranacionales (como «hispanos» o «asiáticos»), insistiendo en las habilidades desarrolladas por estos sujetos para transitar por uno u otro tipo de categorización en función del contexto en que deban definirse. En definitiva, lo que se aprecia en estos casos es que este proceso (que se conoce como etnificación o etnogénesis, y que podría aplicarse igualmente a la identificación nacional) es más resultado de la aceptación o reacción a un etiquetaje externo que resultado del desarrollo endógeno e individual de un sentimiento.

Aproximadamente en la quinta parte de las entrevistas realizadas a adolescentes de segunda generación y de generación intermedia la cuestión de la identificación nacional no fue abordada con claridad, ni siquiera para declarar un sentimiento mixto o combinado, las dudas permanecieron irresueltas o bien el tema fue rehuido por el entrevistado. El análisis de las restantes en que sí se trató la cuestión se ha basado en una clasificación de acuerdo con tres posiciones: la que se identifica con una pertenencia nacional a España, la que niega esta pertenencia y la que opta por una pertenencia múltiple o combinada. Este tipo de clasificación tiene precedentes en otros como el sugerido por Suárez-Orozco y Suárez-Orozco (2003) al distinguir entre identidades de "huida étnica», "oposición activa» $\mathrm{y}$ «transculturales». A la vista de los resultados de esos últimos (que encontraron en este último tipo de identidad el caso más frecuente entre los hijos de inmigrantes) y de la literatura sobre las identidades que ha hecho hincapié en la presencia de los fenómenos que hemos llamado de la «familia de la hibridación» (Terrén, 2007b), la hipótesis esperable era que el tipo de pertenencia que hemos descrito como «múltiple o combinada» fuera la más frecuente.

Hemos encontrado casos, efectivamente, que confirman esta opción. Un joven trabajador de familia marroquí llegado a España en su preadolescencia, por ejemplo, nos decía:

Mira, en un trabajo que he estado en T..., me llamaban «dos sangres», porque decían que tenía dos sangres, la marroquí y la española. Por un lado, yo soy marroquí, pero creo que seré las dos cosas. Me tira España y me tira Marruecos.

Sin embargo, ésta ha sido una posición muy minoritaria. Aunque el hecho de que la selección de nuestra muestra no siguiera ningún criterio 
de representatividad estadística, limita obviamente el valor inferencial de cualquier cuantificación fundamentada en nuestra base de entrevistas. De acuerdo con el carácter exploratorio de nuestra aproximación a la cuestión, vale decir que escasamente una sexta parte (aproximadamente un 15\%) de los hijos de inmigrantes entrevistados exhibieron una pertenencia múltiple de este tipo. Las otras dos opciones de pertenencia definida (bien con el país de origen propio o el de los padres, bien con España) se distribuyeron de la siguiente forma: aproximadamente el 65\% de nuestros entrevistados declararon no sentirse españoles, frente a un $20 \%$ que sí se sentía español.

¿Cómo explicar la masiva presencia de este sentimiento de pertenencia no múltiple y, además, de rechazo hacia la nacionalidad del país de acogida? Una de las hipótesis que albergábamos desde el inicio de la investigación es que el estatus generacional (derivado a partir del momento de llegada) fuera más significativo que el lugar de la procedencia propia o familiar. De nuevo, el carácter de nuestra muestra y el hecho concreto de la sobrerrepresentación en ella de los adolescentes de generación intermedia $(1,5)$ obliga a ser muy prudentes a la hora de extraer generalizaciones, pero el hecho claro encontrado es que algo más de dos terceras partes de los que no se sienten en absoluto españoles son adolescentes de generación 1,5 (así como son también de esta generación dos terceras partes de los muchos menos que expresan un sentimiento combinado de pertenencia, siendo menos de la mitad de los que sí se sienten españoles). Dicho de otra forma, comparativamente (y teniendo en cuenta que nuestra base de segunda generación contempla casi la mitad de casos que la de generación 1,5) el sentimiento de no pertenencia nacional a España es mayoritario, tanto entre los adolescentes de 1,5 generación como en los de segunda (los nacidos aquí), aunque en una proporción mucho menor entre estos últimos (sólo algo más de la mitad). Análogamente, el sentimiento de identificación con España es mayor entre los adolescentes de segunda generación que entre los de generación 1,5 . El sentimiento de pertenencia múltiple, que se esperaba mayoritario, se encuentra menos que la identificación con el origen o con España entre los adolescentes de segunda generación y, aunque poco, es más frecuente que la identificación con España entre los de generación 1,5 .

Los adolescentes más representados en nuestra base de entrevistados fueron los procedentes de familias latinoamericanas (casi la mitad del total). Curiosamente - $\mathrm{O}$ al menos de forma algo inesperada por las habituales expectativas de proximidad que genera el compartir una lengua similar-, casi dos terceras partes (un 64\%) de estos jóvenes, la mayoría de ellos de generación 1,5, no se sienten españoles y, a pesar de que la proximidad cultural o lingüística podría haber hecho esperar una mayor facilidad para la biculturalidad o la pertenencia múltiple, ésta aparece tan poco expresada como en el conjunto (13\%). El segundo grupo más representado según origen en nuestra base fueron los marroquíes (29), la mitad de los cuales (un $53 \%$ ) también se inclinan por el rechazo al sentimiento de pertenencia espa- 
ñola, si bien en menor medida que los adolescentes procedentes de familias latinoamericanas ${ }^{7}$.

En consecuencia, los responsables de que, en los términos generales de nuestra base de entrevistas, prácticamente las dos terceras partes de los adolescentes que se pronuncian sobre la cuestión lo hagan en términos de rechazo a la identificación con España, son los procedentes de las familias latinoamericanas de generación 1,5. La diferencia de casos entre éste y otros grupos de origen no permite comparaciones fiables, pero sí una exploración de los factores que pueden influir en este resultado.

A la hora de estudiar qué es lo que puede hacer tan mayoritario, en la experiencia de los adolescentes traídos a España a partir de los diez años, el rechazo de la identificación con España, hemos rastreado dos hipótesis relacionadas con sus posibles trayectorias de llegada. Una hace referencia a la percepción de la acogida. Otra, a la percepción de los costes del traslado familiar. Veámoslas más en detalle.

La primera hipótesis barajada consiste en suponer que aquellos que han experimentado una situación de discriminación o rechazo (real o percibida) tras su llegada, deberían mostrar un mayor rechazo con mayor probabilidad. Esta asociación era esperada, pues existe en la literatura documentación suficiente de los peculiares efectos asimiladores que tienen las barreras, tanto laborales como institucionales y de convivencia. En nuestro caso, sin embargo, esta variable no parece ser especialmente influyente, pues, entre los adolescentes de 1,5 generación que rechazan el sentirse españoles, los relatos biográficos que contienen episodios de discriminación son tantos como los que no dan cuenta de episodios de este tipo (y el mismo reparto equilibrado se aprecia entre los muchos menos que muestran una pertenencia múltiple). Así es que no parece que el haber experimentado alguna situación de discriminación en carne propia sea muy influyente en la dirección del sentimiento de pertenencia. El caso de un hijo de dominicanos de diecinueve años y llegado a España con cinco es ilustrativo de este punto, pues, a pesar de narrar un incidente en el que fue víctima de racismo institucional por parte de la policía y de declarar haber sido víctima de insultos por el hecho de ser negro, se define como

[...] más español que dominicano, porque yo cuando llegué tenía cinco años y tengo quince en España. Que me considero español a veces ¡̇sabes? Aunque otra gente no lo considere así, yo me considero y mis amigos también me consideran así.

7. El pequeño número de casos correspondientes a otras procedencias geográficas hace muy difícil conceder representatividad, siquiera aproximativa o exploratoria, a los resultados encontrados, pero el hecho es que cinco de los ocho adolescentes entrevistados procedentes de familias de Europa del Este manifestaron ese mismo rechazo a la identificación con España, frente a sólo dos de los siete procedentes de familias subsaharianas (que, sin embargo no registraron ningún caso de identificación) y frente a los cuatro procedentes de familias asiáticas (que sólo registraron una manifestación a favor de la pertenencia española y ninguno en contra de ella). 
Se esperaba también que la relación con la experiencia biográfica de discriminación estuviera asociada a la actividad desempeñada, pues los que a esta edad trabajan, lo hacen en puestos muy precarios y en unas condiciones que hacían muy plausible el suponer que se habrían enfrentado a unas situaciones de discriminación más severas que las que viven quienes a esta edad siguen estudiando. Sin embargo, no hemos podido apreciar esta relación. Contra lo que pudiera esperarse, y con la prudencia a que obliga el que nuestra base cuente con doble número de estudiantes que de trabajadores, parece que los que estudian rechazan algo más la identificación española que los que trabajan.

A la vista de ello, hemos considerado una segunda relación que tiene que ver con otro tipo de experiencia biográfica, más familiar que individual en este caso. Los efectos desestructuradores y estresantes de las rupturas familiares como consecuencia de los traslados migratorios está ya documentada por la investigación (Terrén y Carrasco, 2007; Suárez-Orozco y Suárez-Orozco, 2003: 120-127), y por eso se pensó en la hipótesis consistente en suponer que los adolescentes que han experimentado un proceso traumático de separación familiar reaccionaran contra él, manifestando una identidad de oposición que proyectara sobre el sentimiento de pertenencia el rechazo al proyecto de los progenitores. Esta hipótesis sí parece viable, pues en casi dos terceras partes de los casos en que no existe identificación con España, las narraciones biográficas muestran una acusada experiencia de haber vivido con muchas dificultades la separación a que dio lugar el movimiento por tiempos de la familia.

Por otro lado, el hecho de que los dos grupos más representados en nuestra base de entrevistas hayan sido adolescentes procedentes de un trasfondo cultural y religioso tan distinto (Latinoamérica y Marruecos) obliga a tomar en consideración la posible influencia de la orientación religiosa de las familias. Existen ya investigaciones que han constatado la escasa identificación con la nacionalidad de destino que muestran los inmigrantes de adscripción religiosa musulmana. La encuesta internacional explotada por Álvarez-Miranda (2007), por ejemplo, muestra que, en términos generales, los sentimientos de identidad nacional de los inmigrantes musulmanes en Europa les vinculan más a su país de origen que al de acogida. Sólo la tercera parte de los marroquíes españoles allí encuestados declara sentirse español, y son, con diferencia, el grupo de musulmanes que más siente el orgullo de ser —en su caso- marroquí. Las familias marroquíes de la mayor parte de nuestros entrevistados llevaban en España bastantes años ${ }^{8}$, pero incluso lo dicho por una estudiante de segunda generación hija de matrimonio mixto puede resultar ilustrativo de cómo, aún reconociendo una identificación nacional española, la identificación religiosa es preferente:

8. La tesis clásica viene a decir que estas vinculaciones étnicas o religiosas se debilitan con el tiempo, pero, a falta de estudios longitudinales que lo muestren, no sabemos si la mayor preferencia por las identidades de origen de los llegados más recientemente se debe realmente a su menor tiempo de permanencia o al hecho de pertenecer a una oleada migratoria distinta. 
Antes..., antes musulmana. Claro, porque es mi religión. Y española, pues española porque he nacido aquí y me he criado aquí y siempre seré española, pero musulmana en primero.

Y, de hecho, aun manifestándose por una completa libertad religiosa y de expresión y queriendo ir a la universidad y casarse con quien quiera, declara no poder casarse con un no musulmán. La claridad con que esta chica se define como musulmana no se corresponde con lo difuso con que, en varias ocasiones, intenta autoadscribirse una identidad territorial, como cuando dice:

Yo soy de Madrid, vivo en Madrid y me he quedado en Madrid, pero he nacido en Ceuta y mi origen es de Marruecos.

Este entretejimiento de identificaciones sugiere dos cosas: por un lado, que las asociaciones morales y culturales que los descendientes de musulmanes establecen con la religión, pueden ser recogidas por los procedentes de familias latinoamericanas en una pertenencia nacional o supranacional (como cuando, en repetidas ocasiones, nos han hablado como «latinos» asociándose con cierta forma de conducirse, por ejemplo: en el ocio); por otro, que muchas veces nación y religión (Marruecos e islam) aparecen mezclados en el discurso, como lo prueba una explicación de la misma estudiante al referirse a los atentados de Oriente Próximo e Irak:

[...] en Palestina, en Irak y en el Líbano ahora, pues ellos pues entregan su vida por su patria, por su país, por el islam. También, por ejemplo, con los coches bomba y todo eso..., son unos musulmanes. La gente piensa que son actos terroristas los de Irak, por ejemplo, los del Líbano, pero no, ellos lo hacen porque están defendiendo su país.

Por último, merece la pena rastrear si la expresión del sentimiento de pertenencia se produce de forma diferente en chicos y chicas. La hipótesis esperada es que se diera de forma más definida en los chicos porque existe cierta evidencia de que así sea. Beal (1994), por ejemplo, explicó esa relación por el mayor interés que muestran los chicos por el deporte, lo que supuestamente les mueve a desarrollar una más intensa identidad colectiva. Sin embargo, las entrevistas recogidas en nuestra base no permiten corroborar este punto. La proporción de chicos y de chicas que se posicionan sobre su sentimiento de pertenencia nacional es la misma y, en el conjunto, además, las posiciones están distribuidas con bastante equilibrio, aunque se observa un ligeramente mayor sentimiento de rechazo a la identificación española en las chicas y algunos casos más de pertenencia múltiple en los chicos. Ha llamado la atención que, en diez de las diecisiete entrevistas de chicas de segunda generación (donde la identificación con la nacionalidad del país de acogida parecía más esperable), lo que se haya producido es un rechazo de dicha identificación. 


\section{Conclusiones provisionales}

El estudio de la forma en que los hijos e hijas procedentes de familias inmigradas a España manifiestan sus sentimientos de pertenencia en el curso de una situación de entrevista orientada sobre un modelo de reconstrucción autobiográfico, muestra algunos resultados esperados y otros contrarios a lo que parecía más probable. Aunque, como se ha señalado, la naturaleza de nuestra base de entrevistas no permite tratarla como una muestra estadísticamente representativa de la población estudiada y aunque, por consiguiente, las comparaciones entre categorías deben relativizarse porque, en ocasiones, se comparan grupos de magnitud muy escasa, a partir de las narrativas producidas pueden establecerse algunas afirmaciones de interés exploratorio.

Prácticamente en todos los casos analizados se exhibe inicialmente una gran indefinición y falta de decisión con independencia de la edad. Esto significa que este tipo de identificación no constituye una preocupación central de los adolescentes, pues a lo que hemos asistido prácticamente en todos los casos analizados no es a la manifestación de una preferencia ya asumida, sino a la construcción discursiva de una opción identificacional solicitada. Si es correcta la hipótesis de que hacen falta situaciones desencadenantes que pongan en marcha este proceso de autoidentificación, lo que se infiere es que la inmensa mayoría de nuestros adolescentes procedentes de familias inmigradas no se han sentido expuestos a una situación colectiva lo suficientemente intensa como para forzar con anterioridad el proceso de identificación y elaboración de su pertencia.

Una vez puesto artificialmente en marcha a través de la entrevista, no obstante, casi ocho de cada diez adolescentes procedentes de familias inmigradas terminan mostrando una posición definida respecto a su sentimiento de pertenencia nacional. Una de las hipótesis barajadas a partir de lo que hemos descrito como el straight-model de la asimilación cultural podría haber sido que la segunda generación (por el hecho de haberse criado aquí desde el nacimiento) se mostrara más identificada con la nacionalidad del país de acogida que la generación intermedia. Pero la revisión en la literatura de la crítica a este modelo nos hacía esperar, más bien, que lo que realmente habíamos de encontrar eran muchos casos de lo que suele describirse como identidades transculturales, mixtas o múltiples. No hemos podido corroborar ninguna de las dos hipótesis, puesto que ambas opciones se nos han manifestado claramente minoritarias en relación con quienes muestran un decidido sentimiento de rechazo a la identificación con España. Dos terceras partes de los adolescentes procedentes de familias inmigradas expresan su sentimiento de pertenencia rechazando la identificación con España, y el tercio restante se reparte entre quienes se sienten españoles (o más españoles que) y quienes muestran un sentimiento de pertenencia mixto, sin decantarse expresamente por una de las dos opciones. Los registros encontrados apuntan también a que tanto la opción ampliamente mayoritaria de la preferencia por el origen como la más minoritaria de la identidad múltiple son más frecuentes en la generación intermedia que en la 
segunda, y en ésta sólo es muy ligeramente superior (aunque igualmente minoritaria) la identificación con España. En ninguno de los casos de conformación de identidades mixtas o múltiples, denominadas hyphenated en la literatura sobre las segundas y terceras generaciones, se aprecian expresiones compuestas que manifiestan el reconocimiento formal a una doble pertenencia. Esto quizá pueda explicarse por lo todavía reciente del fenómeno.

La composición de nuestra base de entrevistas impide pronunciarse con rigor cuantitativo sobre la comparación entre procedencias y obliga a reconocer que la dirección general de nuestros resultados se debe a lo expresado por el caso más representado: los adolescentes de generación intermedia procedentes de familias latinoamericanas, algo que no era esperado habida cuenta de la cercanía cultural que habitualmente suele atribuirse a este colectivo. (Es de recordar, sin embargo, que, a pesar de esta cercanía, sus miembros son los únicos que suelen celebrar con cierto carácter institucional los días nacionales de sus países respectivos.) Más que la experiencia de discriminación que se esperaba pudiera explicar el rechazo a la identificación con España, parece ser la ruptura familiar experimentada durante el traslado por tiempos de la familia lo que explica ese rechazo a identificarse con la pertenencia al destino. El amplio rechazo a sentirse españoles parece ser, pues, en buena medida, el rechazo a un proyecto de movilidad y reestructuración familiar que se ha vivido como algo ajeno y estresante.

\section{Referencias bibliográficas}

Álvarez-Miranda, B. (2007). Here and there: Transnational and community links between Muslim immigrants in Europe. Madrid: Real Instituto Elcano WP 9/2007.

ANDERSON, B. (1983). Imagined Communities: Reflections on the Origin and Spread of Nationalism. Nueva York: Verso.

BARRETT, M. (2001). «The development of national identity: a conceptual analysis and some data from Western European studies». En: BarReTt, M.; RiazANOVA, T. y Volovikova, M. (eds.). Development of National, Ethnolinguistic and Religious Identities in Children and Adolescents. Moscú: IPRAS, 16-58.

BarRetT, R.; Riazanova, T. y VoloviKova, M. (eds.) (2001). Development of National, Ethnolinguistic and Religious Identities in Children and Adolescents. Moscú: Institute of Psychology, Russian Academy of Sciences (IPRAS).

BAUBÖCK, R. (1994). Transnational Citizenship: Membership and Rights in International Migration. Aldershot: Elgar.

- (2004). «Cómo transforma la inmigración a la ciudadanía». En: Aubarell, G. y ZAPATA, R. (eds.). Inmigración y procesos de cambio. Barcelona, Icaria, 197-214.

- (2006). «Migración y ciudadanía». Zona Abierta, 116/117, 135-169.

BENHABID, S. (2005). Los derechos de los otros. Extranjeros, residentes y ciudadanos. Barcelona: Gedisa.

Chamberlayne, P.; Bornat, J. y Wengraf, T. (eds.) (2000). The turn to biographical methods in social science: Comparative issues and examples. Londres: Routledge.

Castles, S. y Davidson, A. (2000). Citizenship and Migration: Globalization and the Politics of Belonging. Nueva York: Routledge.

ERIKSON, E. (1992). Identidad. Juventud y crisis. Madrid: Taurus, 1968. 
FAIST, Th. (2004). «Towards a Political Sociology of Transnationalism: The State of the Art in Migration Research». European Journal of Sociology, 45 (3), 19-54.

Fukuyama, F. (2007). "Identity and migration». Prospect Magazine, 131.

García Borrego, I. (2003). "Los hijos de inmigrantes extranjeros como objeto de estudio de la sociología». Anduli: Revista Andaluza de Ciencias Sociales, 3, 27-46.

Glick SCHIller, N. y Fouron, G. (2001). «The Generation of Identity: Redefining the Second Generation Within A Transnational Social Field». En: The Changing Face of Home: The Transnational Lives of the Second Generation. Nueva York: Russell Sage Publications.

Goodenow, C. y EsPIN, O.M. (1993). «Identity Choices in Immigrant Adolescent Females». Adolescence, 28.

Gordon, M.M. (1964). Assimilation in American life. Nueva York: Oxford University Press.

Gusteren, H. van (1994). "Four conceptions of citizenship». En: STEENBERGEN, B. van (ed.). The Condition of citizenship. Londres: Sage, 36-48.

JAHODA, G. (1964). "Children's concepts of nationality: a critical study of Piaget's stages». Child Development, 35, 1081-1092.

Kibria, N. (2002). Becoming Asian American: Second Generation Chinese and Korean American Identities. Baltimore: John Hopkins University Press.

KymlickA, W. (1995). Multicultural Citizenship: A Liberal Theory of Minority Rights. Oxford: Clarendon.

LeVITT, P. (2003). «Keeping Feet in Both Worlds: Transnational Practices and Immigrant Incorporation in the United States». En: JOPPKE, Christian y MORAWSKA, Eva (eds.). Toward Assimilation and Citizenship: Immigrants in Liberal Nation-States. Nueva York: Palgrave Macmillan.

LevitT, P. y Glick Schiller, N. (2004). "Conceptualizing Simultaneity: A Transnational Social Field Perspective on Society». International Migration Review, 38 (3), 1002-1039.

LeVITT, P. y Waters, M. (eds.) (2002). The Changing Face of Home: The Transnational Lives of the Second Generation. Nueva York: Russell Sage Publications.

LUCAS, J. (2001). «Ciudadanía y Unión Europea intercultural». En: AAVV. "Ciudadanía e interculturalidad». Anthropos, 191, 93-116.

LYONS, E. (1996). "Coping with social change: processes of social memory in the reconstruction of identities». En: BREAKWELL, G.M. y LYONS, E. (eds.). Changing European Identities: Social Psychological Analyses of Social Change. Oxford: Butterworth Heinemann.

Martiniello, M. (1994). "Citizenship of the European Union: a critical view». En: Baubock, R. (ed.). From Aliens to Citizens. Aldershot: Avebury Press, 29-47.

Middleton, M.; Tajfel, H. y JoHnson, N. (1970). "Cognitive and affective aspects of children's national attitudes». British Journal of Social and Clinical Psychology, 9, 122-134.

Miller, D. (1997). On Nationality. Oxford: Clarendon Press.

- (2000). Citizenship and National Identity. Cambridge: Polity Press.

Molero, B. (1999). El proceso de construcción infantil de la identidad nacional: Conocimiento del propio pais y de los símbolos nacionales. Servicio editorial de la $\mathrm{UPV} / \mathrm{EHU}$.

MoraWsKA, E. (2003). "Immigrant Transnationalism and Assimilation: A Variety of Combinations and the Analytic Strategy it Suggests». En: JOPPKE, C. y MORAWSKA, E. (eds.). Toward Assimilation and Citizenship: Immigrants in Liberal Nation-States. Nueva York: Palgrave Macmillan. 
- (2004). «Exploring Diversity in Immigrant Assimilation and Transnationalism: Poles and Russian Jews in Philadelphia». International Migration Review, 38 (4), 1372-1405.

Morris, L. (2002). Managing Migration. Civic Stratification and Migrants' Rights. Londres: Routledge.

PHINNEY, J.S. (1990). «Ethnic identity in adolescents and adults: Review of research». Psychological Bulletin, 108 (3), 499-514.

PiagET, J. y WeIL, A. (1951). «The development in children of the idea of the homeland and of relations with other countries». International Social Science Bulletin, 3, 561-576.

Poole, R. (1999). Nation and Identity. Londres/Nueva York: Routledge.

PORTES, A. (2003). «Conclusion: Theoretical Convergences and Empirical Evidence in the Study of Migrant Transnationalism». International Migration Review, 37 (3), 874-892.

PORTES, A. y MACLEOD, D. (1996). «Educational progress of children of immigrants: The roles of class, ethnicity and school context». Sociology of Education, 69, 255-275.

PORTES, A.; GuARNizO, L. y LANDOLT, P. (1999). «Introduction: Pitfalls and Promise of an Emergent Research Field». Ethnic and Racial Studies, 22, 463-478.

REX, J. (1996). «National Identity in the Democratic Multi-Cultural State». Sociological Research Online, 1 (2). [http://www.socresonline.org.uk/socresonline/1/2/1.html]

Riemann, G. (2003). «A Joint Project Against the Backdrop of a Research Tradition: An Introduction to "Doing Biographical Research"». Forum: QualitativeSozialforschung, 4 (3), art. 18. [http://www.qualitative-research.net/fqstexte/]

Rumbaut, R. (1994). «The Crucible Within: Ethnic Identity, Self-Esteem, and Segmented Assimilation Among Children of Immigrants». International Migration Review, 28 (4), 748-94.

SCHWARTZ, S.J.; MONTGOMERY, M.J. y BRIONES, E. (2006). «The Role of Identity in Acculturation among Immigrant People: Theoretical Propositions, Empirical Questions, and Applied Recommendations». Human Development, 49, 1-30.

SEGURA, G.M. (2006). «Immigration and National Identity». Perspectives on politics, 4 (2), junio. APSA.

SOYSAL, Y.N. (1994). Limits of Citizenship: Migrants and Postnational Membership in Europe. Chicago: University of Chicago Press.

SPINNER-Halev, J. y Theiss-Morse, E. (2003). «National Identity and Self-Esteem». Perspectives on Politics, 1, 515-532.

SuÁrez-Orozco, M. y SuÁrez-Orozco, C. (2003). La infancia de la inmigración. Madrid: Morata.

TERRÉN, E. (2003). «Eduación democrática y ciudadanía multicultural: el reaprendizaje de la convivencia». En: BENEDICTO, J. y MORÁN, M.L. (eds.). Aprendiendo a ser ciudadanos. Madrid: INJUVE.

- (2007a). «Interculturalidad, diversidad cultural y globalización». En SUBIRATS, J. y Alegre, J. (coords.). Educación e inmigración: nuevos retos para España en una perspectiva comparada. Madrid: CIS.

- (2007b). «Adolescencia, identidad e inmigración». En: CACHÓN, L. y SALAS, A. (coords.). Juventud e inmigración: Desafios para la participación y la integración. Gobierno de Canarias.

TERRÉN, E. y CARRASCO, C. (2007). «Faith in school: immigrant families' attitudes towards education in Spain». International Studies in Sociology of Education, 17 (4). 
TocQueville, A. (1980). La democracia en América. Madrid: Alianza, 2 vols.

TORRES, E. (1994). «La construcción psicológica de la nación: El desarrollo de las ideas y sentimientos nacionales». En: RODRIGO, M.J. Contexto y desarrollo social. Madrid: Síntesis Psicológica, 305-344.

Turner, T. (1993). "Anthropology and Multiculturalism: what is anthropology that multiculturalists should be mindful of it?». Cultural Anthropology, 8, 411-29.

VELASCO, J.C. (2006): «La desnacionalización de la ciudadanía». En: CAMPOY, I. (ed.). Una discusión sobre la universalidad de los derechos humanos y la inmigración. Dykinson / Universidad Carlos III.

Vertovec, S. (2003). «Migration and Other Modes of Transnationalism: Towards Conceptual Cross-Fertilization». International Migration Review, 37, 641-665.

Vila, I.; Valle, A. del; Perera, S.; Monreal, P. y Barrett, M. (1998). "Autocategorizacion, identidad nacional y contexto lingüístico». Estudios de Psicologia, 60, 3-14.

WATERS, M. (1996). "Ethnic and Racial Identities of Second-Generation Black Immigrants in New York City». En: PORTES, A. (ed.). The New Second Generation. Nueva York: Russell Sage Foundation, 171-96.

- (1999). Black Identities: West Indian Immigrant Dreams and American Realities. Nueva York: Russell Sage Foundation.

Young, I.M. (1990). Justice and the Politics of Difference. Princeton University Press.

ZHOU, M. (2001). «Straddling Different Worlds: The Acculturation of Vietnamese Refugee Children in San Diego». En: Rumbaut, R. y PORTES, A. (eds.). Ethnicities: Coming of Age in Immigrant America. Nueva York: Russell Sage Foundation Press, 187-227.

Zhou, M. y Bankston, C.L. (1998). Growing up American: How Vietnamese children adapt to life in the United States. Nueva York: Russell Sage Foundation. 